\title{
Alcune idee di Ettore Caporali intorno alle quartiche piane.
}

\author{
(Pubblicate da C. Segre, a Torino.)
}

F

Ira gli scritti postumi di Etrone Caporai. che furon messi alla luce nel volume delle sue Memorie di Geometria (Napoli, Pellerano, 1888) vi sono dei frammenti (pag. 344 e seguenti) sulla teoria delle curve piane del quarto ordine il cui studio può essere ajutato da alcune notizie che il Caponatr stesso mi aveva dato delle sue ricerche in due lettere dell' estate 1885. Era mia intenzione, appena comparve il detto volume, di pubblicare quelle notizie in un lavoro in eui avrei trattato delle quartiche piane appunto nell'ordine d'idee del Caporali e tenendo anche conto di alcune importanti e un po' più antiche ricerche del sig. Rexe le quali seguono pure un analogo indirizzo. Ma essendo sempre stato distratto dall' eseguire questo disegno, pubblico ora, con la speranza d'invogliare ed ajutare altri a compiere un tal lavoro, le due lettere del Caporali, od almeno quelle parti di esse che presentano un interesse scientifico, accompagnandole solo con brevi commenti (*).

I.a 1. ${ }^{\mathrm{a}}$ lettera, datata da Torre del Greco 11 agosto 1885 dice:

... “ Ella mi domandò una volta di certe ricerche sulle curve del $4 .^{\circ}$ or"dine che jo avevo intraprese $(* *)$. Ci tenevo assai a quelle povere ricerche, " ma sono da 8 o 9 mesi interrotte affatto. Eccone il concetto.

"Siano dati un sistema lineare $S$ di coniche, di $k$ dimensioni, e, in "questo, un sistema quadratico $\Sigma$ di $k-1$ dimensioni. Fra le coniche di $S$ " e i sistemi lineari di $k-1$ dimensioni (pure di $S$ ) si può stabilire la po" larità rispetto a $\Sigma$. Preso un punto $P$ qualunque del piano, per esso passa " un sistema $\infty^{k-1}$ di coniche di $S$, al quale corrisponde per reciprocità ri-

(*) Il sig. Wirtinger nelle sue Untersuchungen ïber Abel' sche Functionen vom Geschlechte 3 comparse ora nei Math. Annalen (tom. 40, pag. 261) si riferisce ripetutamente ai frammenti del CAPoral sulle quartiche piane ed adopera per lo studio di queste dalle considerazioni iperspaziali identiche in sostanza a quelle che accennerò qui appresso.

$\left.{ }^{* *}\right)$ Di queste ricerche egli $m^{\prime}$ aveva parlato brevemente a Torino un anno avanti. Amali di Matematica, tomo XX. 
" spetto a $\Sigma$ una conica $C$ (di $S$ ). Cos̀ ad ogni punto $P$ è congiunta una " conica $C$. Il luogo dei punti pei quali passano le coniche congiunte è una " curva del $4 .^{\circ}$ ordine $f$. Si hanno cosi varie generazioni della curva generale " del $4 .^{\circ}$ ordine, nelle quali si possono far rientrare molte di quelle conoseiute, " per es. quella per fasei projettivi di coniche. La generazione suindicata si può " enunciare più semplicemente in certi casi. Cosi, per $k=2$ la $f$ non è altro " che l'inviluppo del sistema $\Sigma$. Per $k=3$ il sistema $\Sigma$ contiene due serie $\infty$ " " di fasci di coniche; e la $f$ è simultaneamente il luogo dei punti base dei "fasci delle due serie: due fasci della stessa serie sono projettivi e la generano.

"Tutte le ricerche conosciute intorno alla surva biquadratica rientrano " in quest' ordine d'idee e vi pigliano sistema. Così, quando la curva si può " rappresentare mediante la somma di $k+1$ biquadrati (ossia possiede $\infty^{4-\vec{k}}$ " coniche apolari) fra le infinite generazioni suddette ve n'è una nella quale " la conica congiunta ad un punto è la sua conica polare rispetto alla $f$ stessa. " Io ho studiato particolarmente il caso di $k=3$, nel quale la $f$ possiede una " schiera di coniche apolari e il covariante $S$ si riduce a quattro rette. Vi " sono bellissime proprietà. Per es. la curva è generata da due serie $\infty^{1}$ di " quadrangoli, $\mathrm{i}$ cui lati segano $f$ armonicamente e $\mathrm{i}$ cui vertici descrivono "la hessiana di $f$.

"Se inoltre l'invariante cubico di $f$ si annulla, allora la curva diviene " interessantissima. I suoi flessi si dividono in due gruppi di 12 punti cia" scuno e ogni gruppo è formato dai vertici di quattro trilateri sizigetici. Łे " la curva della quale accennai l'esistenza due anni fa in una breve Nota e " della quale ho poi trovate altre interessanti proprietà.

"Ma, come le ho detto, ho da molto tempo interrotto. Inutile dire che " mi giovaro moltissimo delle considerazioni sugli spazi di $3,4,5$ dimensioni " riferiti projettivamente ai sistemi lineari di coniche. Avevo anche pensato " un po' allo studio analogo dei sistemi quadratici di quadriche, per dedurne " una classificazione projettiva delle superficie del $4 .^{\circ}$ ordine ..."

Quanto allo studio qui accennato dei sistemi quadratici di quadriche, e delle superficie del $4 .^{\circ}$ ordine in relazione con essi, esso era già stato avviato (cosa che pare non fosse nota al $\mathrm{C}_{A P O R A L I}$ ) dal sig. ReYE, specialmente nella Memoria, datata dal 1876 , Ueber die reciproke Verwandtschaft von $F^{2}$-Systemen und $\Phi^{2}$-Geweben und die quadratischen $F^{2}$-Systeme achter Stufe (Journal für Math., tom. 82, pag. 173); lavoro ricco di considerazioni nuove e feconde, da collocarsi, insieme con altri precedenti dello stesso Autore, fra i moderni 
lavori di geometria projettiva a più dimensioni: elementi o punti delle varietà di cui esso tratta essendo le quadriche (dello spazio ordinario).

Appunto in considerazioni della stessa natura si trova la ragione dei principali fatti noti relativi alle quartiche piane, e di quelli annunziati dal Caporali. Invero poichè essi si riferiscono quasi sempre a relazioni tra una quartica e delle coniche (in particolare delle coppie di rette), è naturale di ricorrere per studiarli a quella superficie omaloide normale del $4 .^{\circ}$ ordine $F_{2}^{4}$ dello spazio a cinque dimensioni $S_{5}$ che è rappresentata sul piano $\pi$ dalle $\infty^{5}$ coniche di questo $\left(^{*}\right)$. Indicando con $x_{1} x_{2} x_{3}$ le coordinate di punti in $\pi$, e con $X_{i k}$ (ove $i, k=1,2,3$ e $X_{i k}==X_{k i}$ ) le sei coordinate in $S_{5}$, la $F_{2}^{4}$ si può intendere riferita al piano $\pi$ mediante le formole: $X_{i k}=x_{i} x_{k}$. Ciò posto l'equazione di una quartica $f(x) \equiv a_{x}^{4}=0$ di $\pi$ si potrà scrivere (in infiniti modi) come un'equazione quadratica tra le $X$; e quindi la quartica $f$ avrà per imagine su $F_{2}^{4}$ una curva $C^{8}$ intersezione di questa superficie con una varietà quadratica $M_{4}^{2}$, e quindi con $\infty^{6}$, poichè $F^{4}{ }_{2}$ sta su $\infty^{5} M_{4}^{2}$. La polarità rispetto ad una qualunque di quelle $\infty^{6}$ varietà quadratiche dà subito origine a quella relazione fra punti di $\pi$ e coniche congiunte (rispetto ad un sistema quadratico $\Sigma$ ) di cui parla il CaporaLI, e da cui trae un modo di generazione della quartica $f$. Se poi quella polarità degenera, pel fatto che la $M^{2}{ }_{4}$ abbia un punto, o retta, o piano, doppio, la generazione si semplifica: corrispondentemente al fatto che il sistema quadratico $\Sigma$ di coniche si riduce ad uno giacente in un sistema lineare $S^{\prime}$ di 4, 3,2 dimensioni, rappresentato dagl' iperpiani passanti pel punto, retta o piano doppio della $M^{2}$. Nell' ultimo caso, gli $\infty^{1}$ jperpiani tangenti a questa varietà quadratica lungo $i$ suoi $\infty^{1}$ spazî $S_{3}$ generatori toccano $C^{8}$ nelle quaterne di punti d'incontro di $F^{4}{ }_{2}$ con questi $S_{3}$; e quindi hanno per imagini su $\pi$ delle coniche quadritangenti ad $f$ : si ottiene cioè la generazione della quartica come inviluppo di una $\infty^{\prime}$ quadratica di coniche. Nel secondo caso invece la $M^{2}{ }_{4}$ contiene due schiere di $\infty^{1}$ spazî $S_{3}$ generatori analoghe a quelle delle generatrici di una quadrica ordinaria (sezione della $M^{2}{ }_{4}$ con un $S_{3}$ che non incontri la retta doppia); ed in corrispondenza si avranno su $f$ due schiere di $\infty^{1}$ quaterne di punti, si che due quaterne qualunque di schiere diverse stanno sempre in una stessa conica (sono residue): donde la generazione della quartica mediante fasci projettivi di coniche, ecc.

(*) Verovese, La supenfeie omaloide nommale ecc., Mem. Acc. Lincei (3) XIX, 1884; Segre, Considerazioni intorno alla geometria delle coniche di un piano ecc. Atti Acc. Torino, $\mathrm{XX}, 1885$. 
Tra le $\infty^{6} M^{2}$ passanti per la $C^{8}$ ve n'è sempre una distinta da tutte le altre, la quale analiticamente è data dall'equazione $a_{x}^{3} a_{y}^{2}=0$, quando vi si ponga $x_{i} x_{k}=y_{i} y_{k}=X_{i k}$, e geometricamente è caratterizzata dall' essere apolare ed armonica (come luogo) a tutte le varietà di $2 .^{a}$ classe iscritte nella $\Phi_{2}^{4}$ che è inviluppo degl'iperpiani tangenti a $F^{4}{ }_{2}$ lungo coniche: 0 , come si dice più brevemente, dall' essere apolare alla $\Phi_{2}^{*}\left(^{*}\right)$. La polarità rispetto ad essa si rispecchia sul piano $\pi$ nella corrispondenza di polarità - fra curve di $2 .^{\circ}$ ordine c curve di $2 .^{\circ}$ classe, ed in particolare fra punti e coniche - rispetto alla quartica $f$, che vien determinata dall' equazione $a_{x}^{2} a_{y}^{2}=0$. Ad una conica apolare rispetto alla quartica corrisponde un punto doppio per la $M_{4}^{2}$; e cosi questa acquista una retta doppia, se la quartica ammette una schiera di coniche apolari; ecc., ecc.

Avendo io comunicato al Caporalı, forse con qualche maggior sviluppo, questo modo con cui vedevo le cose da lui enunciate, - metodo che non è del resto se non un'applicazione particolare di un procedimento molto generale, utile per lo studio di enti svariatissimi, - egli mi rispose in un'altra lettera da Torre del Greco, 13 settembre 1885 quanto segue.

" . . Ciò che Ella mi scrive intorno ai miei studi sulle curve del $4 .^{\circ}$ or"dine è interessante e dimostra che Ella ha immediatamente penetrato lo " spirito di quelle ricerche. Per quanto poco avanzate, esse hanno una storia " complicata $\mathrm{e}$ in relazione con diverse cause estranee alla scienza che m'im" pediscono da tre anni di attendere allo studio con quella regolarità e quella " perseveranza che sole permettono di cavarne buoni frutti.

"Tre o quattro anni fa, studiando le memorie algebriche intorno allo " curve piane del $3 .^{\circ}$ ordine, mi accorsi per caso che il combinante $N=$ " $(a \alpha u) a_{x}^{2} \alpha_{\infty}^{2}$ (dove $a^{3}{ }_{x}=0, \alpha^{3}{ }_{x}=0$ sono le equazioni d'una cubica e della " sua hessiana) rappresenta, nelle variabili $x$, una curva del $4 .^{\circ}$ ordine che " ha per flessi i 12 vertici dei trilateri appartenenti al fascio $a_{x}^{3}+\lambda a_{x}^{3}=0$. "L'esistenza di una rete (i parametri essendo le $u$ ) di curve del $4 .^{\circ}$ ordine " con 12 flessi in comune riusciva per me nuova ed è senza dubbio interes" sante, non è vero? Dimodochè mi posi ad esaminare meglio quella curva " e riconobbi che gli altri 12 flessi formavano una configurazione perfetta" mente analoga dando luogo ad un secondo fascio sizigetico. Pel momento " la cosa rimase li: ma più tardi riportai di nuovo la mia attenzione su quel

(') Per tal modo le $\infty^{14}$ quartiche ael piano $\pi$ sono rappresentate anivocamente dalle $\infty^{\text {it }}$ varietà $M_{4}^{2}$ di $S_{5}$ apolari a $\Phi_{2}^{4}$. 
"fatto nuovo e notevole, che vi siano due fasci sizigetici $i$ quali dànno lo " stesso combinante $N$. E, mentre al principio m'ero servito d'equazioni ca" noniche, volli intraprendere i calcoli simbolici necessarii a dimostrare quella " proprietà e a dedurre dall'uno dei due fasci l'altro. Feci all' Accademia la " comunicazione provvisoria Sopra una certa curva del $4 .^{\circ}$ ordine $\left(^{*}\right)$ e mi posi " al lavoro. Mi trastullai per un pezzo in laboriose calcolazioni (del resto in" teressanti), ma ad un certo punto fui deviato momentaneamente dagli studi " ed ogni cosa rimase ed è rimasta interrotta.

"L'anno scorso, senza riprendere $\mathrm{i}$ calcoli, tornai sulle considerazioni " geometriche e m'accorsi che la curva possedeva una schiera di coniche " apolari, senza però essere la più generale di questa specie. Queste curve, è " facile vederlo, possono studiarsi con successo nel piano rappresentativo della " superficie romana di Sterner. Fu allora che la ricerca comincio ad allar" garsi sino a che gradatamente ha preso l'aspetto attuale. Nelle vacanze " dell'anno scorso cominciai dunque a giovarmi delle considerazioni sugli " spazî di più dimensioni, colle quali ho antica famigliarita. Rifeci le pro" prietà delle tangenti doppie, specialmente per le curve dotate di punti sin" golari: lavoro piuttosto minuto. Iniziai anche lo studio dei flessi colla ricerca " dei sistemi di coniche biosculatrici je chiaro che le coniche di un sistema " riescono biosculatrici al loro inviluppo, quando sono rappresentate dai punti " d'una curva lo cui tangenti incontrano $\left.F^{4}{ }_{2 \cdot}\left({ }^{* *}\right)\right]$. Dovetti però presto so" spendere per malattie di famiglia e non ho più ripreso.

"Quando Ella pubblico la sua memoria sulla geometria delle coniche, " vidi immediatamente il partito che si poteva trarre dall' uso sistematico di " quel modo di rappresentazione e che mi è confermato dalla sua... lettera: " non ripresi pero, nè potrò subito riprendere le ricerche, benchè precisamente " ora, avendo acquistata tutta la loro generalità, siano nello stadio più in" teressante. Lo farò però a novembre, almeno spero.

(***) “ Per cambiare discorso, le parlerò d'un'altra mia piccola Nota in" compiuta che potrà pure interessarla. È notissimo nello spazio ordinario il

(*) Rendic. Ace. Napoli, dicembre 1882.

(**) Ció non è pienamente esatto. Quando la tangente in un punto alla curva rappresentante di un sistema di coniche incontra $F_{*}^{4}$, la conica corrispondente a quel punto ha dre contathi quadripunti coll' inviluppo...

(**:) Quanto segue non riguarda piu le quartiche piane, ma lo riporto qui ugualmente perchè parmi possa pure interessare: trattandosi di una questione importantissima, che, con quel grado di generalità, non si trova nel volume di memorie del Caporazr, né da altri $\dot{e}$ stuta finora risolta. 
“ problema di determinare le singolarità ordinarie d'una curva intersezione " parziale di due superficie quando si conoscano quelle della residuale inter"sezione: e l'altro susseguente di trovare le intersezioni di tre superficie " assorbite da una curva comune. Le notissime soluzioni che se ne leggono " in Sarmon e in Cremona si estendono facilmente in certi casi allo spazio " di $n$ dimensioni: quando cioè si tratta della intersezione parziale di più va" rietà di $n-1$ dimensioni, definite mediante altrettante equazioni generali " nel loro grado. Il Veronese ha trattato il caso più ovvio in cui questa in" tersezione è una curva. Ma quando le varietà che si segano hanno meno " di $n-1$ dimensioni e sono esse stesse intersezioni parziali, il metodo di "Salmon non si può più seguire. Si hanno i primi esempi di questa difficoltà "nello spazio di 4 dimensioni in questi due problemi: 1. Quando la curva " comune ad una varietà e ad una superficie si spezza in due parti, date le “ singolarità dell' una trovare quelle dell'altra. 2. Trovare i punti d'interse" zione di due superficie assorbite da una curva comune.

"Ora io sono pervenuto ad una formola generalissima la quale, quando " sia dato un numero qualunque di varietà ad un numero qualunque di di(6 mensioni, fornisce le singolarità fondamentali della varieta ad esse comune, "se è semplice; orvero le relazioni fra le singolarità delle due parti, se essa " si spezza. Le comunicherei addirittura questa formola, che può essere utile " pel gran numero di casi che abbraccia, se qui in campagna avessi le mie a note o se la memoria mi aiutasse. Essa è complicata, perchè non solo con"tiene gli ordini e le serie di ranghi delle diverse varietà (le quali possono " anche possedere singolarità superiori) ma altri numeri il cui significato offre " molto interesse. Una varieta può essere contenuta in un'altra in diversi " modi, ognuno dei quali è caratterizzato dai valori di certi numeri, valori " che bisogna conoscere per poter risolvere parecchi problemi. Per darle un "esempio, nello spazio ordinario, quando si dice che una curva giace sopra " una superficie dotata di curva doppia, bisogna dare il numero dei punti che " la curva ha in comune colla curva doppia della superficie, numero che, "entro certi limiti, può variare per la stessa curva e la stessa superficie. " Ciò posto, Ella comprenderà facilmente come per una superficie, anche ge" nerale, d'uno spazio di più di 3 dimensioni, ci sia un numero analogo, " poichè essa si proietta nello spazio ordinario in una superficie con curva " doppia. La cosa naturalmente si complica per le altre varietà e la fatica " maggiore l'bo fatta per stabilire questi concetti."

Torino, agosto 1892. 